ISSN 0103-9954

\title{
COMPOSIÇÃO E ABUNDÂNCIA DA AVIFAUNA EM QUATRO FITOFISIONOMIAS DE ÁREA RURAL PERTENCENTE AO DOMÍNIO DA MATA ATLÂNTICA NO SUL DO BRASIL
}

\author{
AVIFAUNA COMPOSITION AND ABUNDANCE IN FOUR VEGETATION PHYSIOGNOMIES OF \\ A RURAL AREA OF THE ATLANTIC FOREST DOMAIN IN SOUTHERN BRAZIL
}

Marcelo Fischer Barcellos dos Santos ${ }^{1}$ Cristina Vargas Cademartori ${ }^{2}$

\begin{abstract}
RESUMO
Estudaram-se a composição e a abundância da avifauna em área rural do município de Viamão, Rio Grande do Sul. A área de estudo consiste de campos utilizados em atividades agropastoris ( $20 \mathrm{ha}$ ), remanescente de floresta secundária de aproximadamente 7 ha, plantio de eucalipto com sub-bosque nativo (6 ha) e plantio misto de Pinus sp. (1 ha). Levantamentos quali-quantitativos (por meio de transecções) foram realizados entre 5 de maio e 20 de novembro de 2006, a partir de visualizações ou das vocalizações emitidas pelas espécies, totalizando-se um esforço de $80 \mathrm{~h}$. Foram registradas 92 espécies de aves na área. Algumas espécies ocorreram exclusivamente na mata secundária, como Crypturellus obsoletus, Piculus aurulentus, Sclerurus scansor, Dendrocolaptes platyrostris, Conopophaga lineata, Myiarchus swainsoni, Chiroxiphia caudata e Euphonia pectoralis, indicando maior seletividade quanto à escolha de habitats. Destacam-se os registros de Piculus aurulentus, espécie que se encontra quase ameaçada de extinção em nível global, e Dendrocolaptes platyrostris, espécie que carece de registros para a região amostrada. Os resultados obtidos demonstram a importância de áreas com vegetação nativa para a manutenção de espécies mais seletivas quanto à escolha de habitats e suscitam a necessidade de novos estudos na área e em fragmentos próximos, com o objetivo de se conhecer melhor a composição e a abundância de espécies em ambientes degradados. Palavras-chave: avifauna; área rural; remanescentes florestais.
\end{abstract}

\begin{abstract}
We studied the avifauna composition and its abundance in a rural area of the city of Viamão, in the state of Rio Grande do Sul, southern Brazil. The study area was composed of fields used for cattle breeding and for agricultural activities (20 ha), secondary forest (7 ha), eucalypt plantation (6 ha) and Pinus sp. plantation (1 ha). Quantitative and qualitative surveys (using line transects) were carried out from May 5 to November 20, 2006. Every detected bird (heard or seen) was considered in the analysis. The survey comprised a total of 80 hours. We recorded 92 species of birds in the study area. Some species were recorded only at the secondary forest, such as Crypturellus obsoletus, Piculus aurulentus, Sclerurus scansor, Dendrocolaptes platyrostris, Conopophaga lineata, Myiarchus swainsoni, Chiroxiphia caudata and Euphonia pectoralis, which indicate more selectivity in certain habitats. We emphasize the occurrences of Piculus aurulentus, an almost endangered species worldwide, and Dendrocolaptes platyrostris, which has no previous record in the region studied. The results show the importance of native forests for the maintenance of more selective bird species and indicate that new studies are necessary in the area and in adjacent forest fragments, in order to improve the knowledge about the composition and the abundance of bird species in degraded environments.
\end{abstract}

Keywords: avifauna; rural area; forest remnants.

1 Biólogo, MSc., Grupo de Pesquisa Meio Ambiente e Impacto Antrópico, Pós-Graduação e Pesquisa, Centro Universitário La Salle, Av. Victor Barreto, 2288, CEP 92010-000, Canoas (RS), Brasil. fischeri_80@hotmail.com

2 Bióloga, Dr ${ }^{\mathrm{a}}$., Professora do Mestrado em Avaliação de Impactos Ambientais, Centro Universitário La Salle, Av. Victor Barreto, 2288, CEP 92010-000, Canoas (RS), Brasil. cristinacademartori@unilasalle.edu.br

Recebido para publicação em 6/09/2012 e aceito em 3/09/2013

Ci. Fl., v. 25, n. 2, abr.-jun., 2015 


\section{INTRODUÇÃO}

A região metropolitana de Porto Alegre, assim como a própria capital gaúcha, sofreu, nas últimas décadas, com a descaracterização e fragmentação dos habitats naturais que forneciam condições apropriadas à sobrevivência de diversas espécies de aves (BELTON, 1994; GRILLO e BENCKE, 1995; MENDONÇA-LIMA e FONTANA, 2000; ACCORDI, 2001; FONTANA, 2005; SANTOS e CADEMARTORI, 2007). Tais alterações ambientais, que geralmente resultam em um complexo mosaico de ilhas de vegetação em meio à malha urbana, podem afetar negativamente muitas espécies (WILLIS, 1979; MATARAZZO-NEUBERGUER, 1995; WILLIS, 2000; ANJOS, 2001; BENCKE et al., 2003; GIMENES e ANJOS, 2003; SCHERER et al., 2005).

A substituição da vegetação original impõe novas condições ecológicas à avifauna, fazendo com que a composição de espécies em determinado local se modifique e passe a incluir táxons mais tolerantes, em detrimento daqueles mais seletivos que demandam requisitos ecológicos específicos para sobreviver (WILLIS, 1979; MATARAZZONEUBERGUER, 1995; SICK e PACHECO, 1997; MENDONÇA-LIMA e FONTANA, 2000; WILLIS, 2000; ACCORDI, 2001; FONTANA, 2005; SANTOS e CADEMARTORI, 2010). Estudos em ambientes urbanos têm demonstrado que inúmeras espécies ocupam áreas verdes no interior das cidades ou em suas adjacências, utilizando esses locais como refúgio, para nidificação e alimentação (VOSS, 1981; SANDER e VOSS 1982; TAMPSON, 1990; MATARAZZO-NEUBERGUER, 1995; MENDONÇA-LIMA e FONTANA, 2000; ACCORDI, 2001; FONTANA, 2005; SCHERER et al., 2006; SANTOS e CADEMARTORI, 2010).

Pesquisas de mais longo prazo ou séries históricas evidenciam, inequivocamente, a perda rápida e significativa de biodiversidade e a homogeneização biótica nas zonas urbanas. Willis (2000) registra, para 300 localidades amostradas no estado de São Paulo, de 1975 a 1998, consideradas impactadas pela ação antrópica, uma lista de 30 espécies com ampla distribuição no estado e consideradas por ele como associadas a ambientes antrópicos. Em Porto Alegre, Fontana (2005), a partir de extenso levantamento bibliográfico no qual reúne todos os registros de aves para o município, conjugado com observações pessoais e de colaboradores, avaliou o estado de conservação das aves na capital gaúcha. Dos 267 registros compilados desde 1930, apenas 169 espécies de aves foram consideradas de ocorrência regular no município e 13 espécies surgiram como colonizadoras.

Considerando a velocidade com que os habitats vêm sendo alterados ou suprimidos, avaliações da avifauna em regiões adjacentes a grandes centros urbanos podem subsidiar programas de conservação e recuperação de áreas degradadas. Neste sentido, teve-se como objetivo, conhecer a composição e a abundância da avifauna, bem como suas variações espaciais, em uma área rural do município de Viamão, contíguo a Porto Alegre, no estado do Rio Grande do Sul.

\section{MATERIAL E MÉTODOS}

\section{Área de estudo}

A área de estudo situa-se próximo ao limite sul de Porto Alegre, na localidade do Lami, entre as coordenadas geográficas de $30^{\circ} 14^{\prime} \mathrm{S}$ e $51^{\circ} 02^{\prime} \mathrm{W}$. Consiste em uma propriedade rural composta por campos antrópicos com atividades agrícolas e de pastagem (20 ha), remanescente de floresta nativa secundária (aproximadamente $7 \mathrm{ha}$ ), plantio de eucalipto (Eucalyptus sp.) com sub-bosque nativo (6 ha), e plantio misto de pinus (Pinus sp.) com vegetação nativa e banhado (1 ha). O entorno é composto por diversas propriedades particulares com atividades diversificadas, especialmente silvicultura e fruticultura. Em alguns locais podem ser encontrados remanescentes da vegetação nativa de dimensões variadas, como fragmentos de floresta secundária pertencentes ao Domínio da Mata Atlântica (mais especificamente, Floresta Estacional Semidecidual) e áreas úmidas.

A floresta nativa secundária apresenta dossel entre 6 e $12 \mathrm{~m}$ com sub-bosque pouco desenvolvido devido a sua utilização eventual como refúgio para o gado. O plantio de eucalipto e o plantio misto de pinus, com mais de 50 anos, apresentam copas com altura aproximada de $20 \mathrm{~m}$. No primeiro, há um sub-bosque denso, uma vez que a área não é utilizada comercialmente para corte. O segundo (que também não é explorado comercialmente) apresenta sub-bosque heterogêneo com diversas espécies arbóreas associadas, além de uma pequena área alagada com vegetação palustre associada. A área aberta caracteriza-se por apresentar constituição heterogênea, incluindo áreas de campo (utilizadas para fins pastoris), árvores 
esparsas, arbustos (geralmente formando vassourais do gênero Baccharis), pomares e cultivos agrícolas de subsistência. Além disso, há também um lago artificial associado a uma pequena área úmida.

\section{Procedimentos de campo e analíticos}

Procedeu-se a avaliações quali-quantitativas na área de estudo, de 5 de maio a 20 de novembro de 2006. Cada local foi amostrado por $20 \mathrm{~h}$, totalizando-se $80 \mathrm{~h}$ de observações. Aárea de campo antrópico foi avaliada apenas qualitativamente. $\mathrm{O}$ remanescente de floresta secundária, o plantio de eucalipto e o plantio misto de pinus foram avaliados tanto qualitativamente quanto quantitativamente.

Utilizou-se a metodologia de transecções de distância ilimitada (WILLIS, 1979) para coleta de dados referentes à abundância de espécies, as quais foram identificadas auditivamente ou visualmente, com o auxílio de um binóculo Konus 8 x 40, sempre pelo mesmo observador (SANTOS, M.F.B.). Todas as espécies avistadas ou ouvidas foram consideradas na análise, desde que estivessem dentro dos limites da área amostrada. As transecções iniciavam nas primeiras horas do dia e estendiam-se até $10 \mathrm{~h}$ da manhã. Durante o período da tarde, entre 15 h e 18 $\mathrm{h}$, foram realizadas caminhadas aleatórias apenas com o objetivo de registrar espécies.

A nomenclatura sistemática adotada está de acordo com o Comitê Brasileiro de Registros Ornitológicos (CBRO, 2010). A classificação de guildas tróficas segue Motta-Junior (1990) e Efe et al. (2001), além de observações pessoais.

Utilizou-se a abundância relativa (calculada separadamente para cada ambiente), a frequência de ocorrência e o Índice de Diversidade de Shannon como descritores da avifauna. A frequência de ocorrência foi obtida a partir da razão entre o número de registros de cada espécie e o número de visitas em cada fitofisionomia estudada. Empregouse o coeficiente de Jaccard para investigar o grau de similaridade entre as áreas no que se refere à composição da avifauna, por meio do programa MVSP (KOVACH, 1999) versão 3.1 para Windows. Fez-se uso do teste do qui-quadrado para avaliar diferenças entre a frequência absoluta de indivíduos entre as distintas áreas amostradas (ANJOS, 2001); os resultados foram considerados significativos para $\mathrm{P}<0,05$. O teste foi executado no programa Systat 11 para Windows (WILKINSON, 2004).

\section{RESULTADOS}

Foram registradas 92 espécies de aves na área de estudo. Destas, 44 foram constatadas na floresta nativa secundária (12 exclusivas), 31 no plantio de eucalipto (uma exclusiva), 38 no plantio misto de pinus (quatro exclusivas) e 57 na área aberta (31 exclusivas), conforme a Tabela 1. A curva cumulativa de espécies (Figura 1) demonstra que houve tendência à estabilização no registro de novas espécies, indicando que o esforço amostral foi suficiente para evidenciar o conjunto da avifauna nos ambientes amostrados.

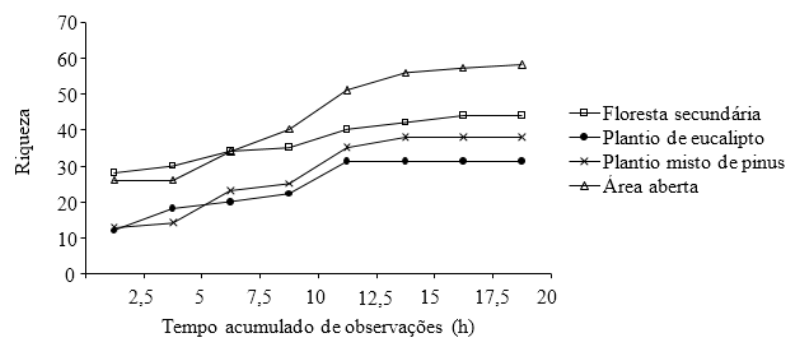

FIGURA 1: Curva cumulativa de espécies de aves registradas em área rural no município de Viamão, Rio Grande do Sul, de maio a novembro de 2006.

FIGURE 1: Cumulative curve of bird species registered in a rural area of the city of Viamão, state of Rio Grande do Sul, from May to November 2006.

O remanescente de floresta secundária foi o ambiente que apresentou a maior frequência absoluta de indivíduos $(\mathrm{n}=193)$ e a maior diversidade específica $\left(\mathrm{H}^{\prime}=3,18\right)$, seguido pelo plantio misto de pinus $\left(\mathrm{n}=52 ; \mathrm{H}^{\prime}=2,93\right)$ e pelo plantio de eucalipto $\left(\mathrm{n}=39 ; \mathrm{H}^{\prime}=2,5\right)$. Houve diferença significativa na frequência absoluta entre as áreas estudadas $\left(\chi^{2}\right.$ $=154,106, \mathrm{gl}=2, \mathrm{P}<0,001) . \mathrm{O}$ remanescente de floresta nativa comportou maior frequência em relação ao plantio de pinus $\left(\chi^{2}=81,147, \mathrm{gl}=1\right.$, $\mathrm{P}<0,001)$ e ao plantio de eucalipto $\left(\chi^{2}=231,804\right.$, $\mathrm{gl}=1, \mathrm{P}<0,001)$. O plantio de pinus e o plantio de eucalipto não apresentaram diferença significativa em relação à frequência absoluta $\left(\chi^{2}=1,857, \mathrm{gl}=1\right.$, $\mathrm{P}=0,173$ ).

A análise de similaridade de Jaccard (Figura 2) mostra clara distinção entre as áreas estudadas quanto à composição de espécies. A área aberta, principalmente, diferenciou-se das demais por apresentar espécies não associadas a ambientes 
TABELA 1: Abundância relativa e frequência de ocorrência (FO) das espécies de aves registradas em quatro fitofisionomias de propriedade rural no município de Viamão - RS, de maio a novembro de 2006. FS = floresta secundária, $\mathrm{PE}=$ plantio de eucalipto, $\mathrm{PP}=$ plantio misto de pinus e $\mathrm{AB}=$ área aberta. $\mathrm{O}$ " $\mathrm{x}$ " indica a presença da espécie quando não se obtiveram dados relativos à abundância.

TABLE 1: Relative abundance and frequency of occurrence (FO) of birds registered in a rural area of the city of Viamão, state of Rio Grande do Sul, from May to November 2006. FS = secondary forest, $\mathrm{PE}=$ Eucalypt plantation, $\mathrm{PP}=$ Pinus sp. plantation and $\mathrm{AB}=$ open area. The " $\mathrm{x}$ " indicates the presence of the species when it was not possible to find data related to abundance.

\begin{tabular}{|c|c|c|c|c|c|c|c|c|}
\hline \multirow{2}{*}{ FAMÍLIA/Espécie } & \multirow{2}{*}{$\begin{array}{c}\text { FS } \\
\text { abundância }\end{array}$} & \multicolumn{3}{|c|}{$\mathrm{PE}$} & \multirow{2}{*}{$\begin{array}{c}\text { PP } \\
\text { abundância }\end{array}$} & \multicolumn{3}{|c|}{$\mathrm{AB}$} \\
\hline & & FO & abundância & FO & & FO & abundância & FO \\
\hline \multicolumn{9}{|l|}{ TINAMIDAE } \\
\hline Crypturellus obsoletus & 0,01 & 0,78 & & & & & & \\
\hline Nothura maculosa & & & & & & & $\mathrm{x}$ & 0,33 \\
\hline \multicolumn{9}{|l|}{ ANATIDAE } \\
\hline Amazonetta brasiliensis & & & & & & & $\mathrm{x}$ & 0,56 \\
\hline \multicolumn{9}{|l|}{ CRACIDAE } \\
\hline Ortalis guttata & 0,01 & 0,67 & $\mathrm{x}$ & 0,22 & $\mathrm{x}$ & 0,11 & & \\
\hline \multicolumn{9}{|l|}{ CICONIIDAE } \\
\hline Mycteria americana & & & & & & & $\mathrm{x}$ & 0,11 \\
\hline \multicolumn{9}{|l|}{ ARDEIDAE } \\
\hline Nycticorax nycticorax & & & & & $\mathrm{x}$ & 0,33 & & \\
\hline Bubulcus ibis & & & & & & & $\mathrm{x}$ & 0,89 \\
\hline Syrigma sibilatrix & & & & & 0,04 & 0,44 & $\mathrm{x}$ & 0,67 \\
\hline \multicolumn{9}{|l|}{ CATHARTIDAE } \\
\hline Cathartes aura & & & & & & & $\mathrm{x}$ & 0,67 \\
\hline Coragyps atratus & & & & & & & $\mathrm{x}$ & 1,00 \\
\hline \multicolumn{9}{|l|}{ ACCIPITRIDAE } \\
\hline Rupornis magnirostris & 0,01 & 0,22 & $\mathrm{x}$ & 0,22 & & & $\mathrm{x}$ & 1,00 \\
\hline \multicolumn{9}{|l|}{ FALCONIDAE } \\
\hline Caracara plancus & & & & & & & $\mathrm{x}$ & 0,44 \\
\hline Milvago chimachima & & & & & 0,02 & 0,67 & $\mathrm{x}$ & 1,00 \\
\hline Milvago chimango & & & & & & & $\mathrm{x}$ & 1,00 \\
\hline \multicolumn{9}{|l|}{ RALLIDAE } \\
\hline Aramides saracura & & & & & 0,04 & 1,00 & & \\
\hline \multicolumn{9}{|l|}{ CHARADRIIDAE } \\
\hline Vanellus chilensis & & & & & & & $\mathrm{x}$ & 1,00 \\
\hline \multicolumn{9}{|l|}{ JACANIDAE } \\
\hline Jacana jacana & & & & & & & $\mathrm{x}$ & 1,00 \\
\hline \multicolumn{9}{|l|}{ COLUMBIDAE } \\
\hline Columbina picui & & & & & $\mathrm{x}$ & 1,00 & $\mathrm{x}$ & 1,00 \\
\hline Patagioenas picazuro & $\mathrm{x}$ & 0,67 & $\mathrm{x}$ & 0,56 & 1,92 & 0,44 & & \\
\hline Zenaida auriculata & & & & & $\mathrm{x}$ & 1,00 & $\mathrm{x}$ & 1,00 \\
\hline Leptotila verreauxi & 0,02 & 1,00 & 0,18 & 1,00 & 0,02 & 0,67 & & \\
\hline Leptotila rufaxilla & 0,01 & 0,56 & 0,03 & 0,44 & & & & \\
\hline
\end{tabular}


TABELA 1: Continuação ...

TABLE 1: Continued ...

\begin{tabular}{|c|c|c|c|c|c|c|c|c|}
\hline \multirow{2}{*}{ FAMÍLIA/Espécie } & \multirow{2}{*}{$\begin{array}{c}\text { FS } \\
\text { abundância }\end{array}$} & \multicolumn{3}{|c|}{$\mathrm{PE}$} & \multirow{2}{*}{$\begin{array}{c}\text { PP } \\
\text { abundância }\end{array}$} & \multicolumn{3}{|c|}{$\mathrm{AB}$} \\
\hline & & FO & abundância & FO & & FO & abundância & FO \\
\hline \multicolumn{9}{|l|}{ PSITTACIDAE } \\
\hline Myiopsitta monachus & & & & & & & $\mathrm{x}$ & 0,56 \\
\hline \multicolumn{9}{|l|}{ CUCULIDAE } \\
\hline Piaya cayana & 0,02 & 1,00 & $\mathrm{x}$ & 0,22 & & & $\mathrm{x}$ & 0,11 \\
\hline Crotophaga ani & & & & & & & $\mathrm{x}$ & 0,56 \\
\hline Guira guira & & & & & & & $\mathrm{x}$ & 1,00 \\
\hline Tapera naevia & & & & & & & $\mathrm{x}$ & 0,22 \\
\hline \multicolumn{9}{|l|}{ TROCHILIDAE } \\
\hline Stephanoxis lalandi & 0,02 & 0,89 & 0,03 & 0,33 & & & & \\
\hline Thalurania glaucopis & & & & & & & $\mathrm{x}$ & 0,22 \\
\hline Hylocharis chysura & $\mathrm{x}$ & 1,00 & 0,05 & 0,44 & $\mathrm{x}$ & 0,78 & $\mathrm{x}$ & 1,00 \\
\hline \multicolumn{9}{|l|}{ PICIDAE } \\
\hline Veniliornis spilogaster & 0,01 & 0,67 & $\mathrm{x}$ & 0,22 & 0,02 & 0,56 & $\mathrm{x}$ & 0,33 \\
\hline Piculus aurulentus & 0,02 & 0,56 & & & & & & \\
\hline Colaptes melanochloros & $\mathrm{x}$ & 0,44 & 0,03 & 0,22 & $\mathrm{x}$ & 0,44 & $\mathrm{x}$ & 0,67 \\
\hline Colaptes campestris & & & & & & & $\mathrm{x}$ & 1,00 \\
\hline \multicolumn{9}{|l|}{ THAMNOPHILIDAE } \\
\hline Thamnophilus caerulescens & 0,02 & 1,00 & $\mathrm{x}$ & 0,78 & & & & \\
\hline Makenziaena leachi & $\mathrm{x}$ & 0,33 & & & & & & \\
\hline \multicolumn{9}{|l|}{ CONOPOPHAGIDAE } \\
\hline Conopophaga lineata & 0,01 & 0,44 & & & & & & \\
\hline \multicolumn{9}{|l|}{ SCLERURIDAE } \\
\hline Sclerurus scansor & 0,03 & 0,67 & & & & & & \\
\hline \multicolumn{9}{|l|}{ DENDROCOLAPTIDAE } \\
\hline Sittasomus griseicapillus & 0,07 & 1,00 & & & 0,02 & 0,33 & & \\
\hline Lepidocolaptes falcinellus & $\mathrm{x}$ & 1,00 & $\mathrm{x}$ & 0,11 & $\mathrm{x}$ & 0,33 & & \\
\hline Dendrocolaptes platyrostris & 0,01 & 0,44 & & & & & & \\
\hline \multicolumn{9}{|l|}{ FURNARIIDAE } \\
\hline Furnarius rufus & & & & & $\mathrm{x}$ & 1,00 & $\mathrm{x}$ & 1,00 \\
\hline Syndactyla rufosuperciliata & 0,04 & 1,00 & 0,03 & 0,56 & 0,02 & 0,44 & & \\
\hline Synallaxis spixi & & & & & & & $\mathrm{x}$ & 0,56 \\
\hline Cranioleuca obsoleta & 0,03 & 1,00 & $\mathrm{x}$ & 0,22 & 0,12 & 1,00 & & \\
\hline \multicolumn{9}{|l|}{ PIPRIDAE } \\
\hline Chiroxiphia caudata & 0,02 & 0,67 & & & & & & \\
\hline \multicolumn{9}{|l|}{ RHYNCHOCYCLIDAE } \\
\hline Phylloscartes ventralis & 0,04 & 0,89 & 0,03 & 0,22 & $\mathrm{x}$ & 0,56 & & \\
\hline Tolmomyas sulphurescens & 0,01 & 0,56 & 0,08 & 0,56 & 0,04 & 0,33 & & \\
\hline Poecilotriccus plumbeiceps & & & 0,08 & 1,00 & & & & \\
\hline \multicolumn{9}{|l|}{ TYRANNIDAE } \\
\hline Camptostoma obsoletum & 0,03 & 0,44 & $\mathrm{x}$ & 0,44 & 0,02 & 1,00 & $\mathrm{x}$ & 1,00 \\
\hline
\end{tabular}

Continua ... 
TABELA 1: Continuação ...

TABLE 1: Continued ...

\begin{tabular}{|c|c|c|c|c|c|c|c|c|}
\hline \multirow{2}{*}{ FAMÍLIA/Espécie } & \multirow{2}{*}{$\frac{\text { FS }}{\text { abundância }}$} & \multicolumn{3}{|c|}{$\mathrm{PE}$} & \multicolumn{2}{|l|}{ PP } & \multicolumn{2}{|l|}{$\mathrm{AB}$} \\
\hline & & FO & abundância & FO & abundância & FO & abundância & FO \\
\hline Elaenia flavogaster & & & $\mathrm{x}$ & 0,33 & & & $\mathrm{x}$ & 0,56 \\
\hline Elaenia parvirostris & $\mathrm{x}$ & 0,56 & $\mathrm{x}$ & 0,33 & $\mathrm{x}$ & 0,44 & $\mathrm{x}$ & 0,33 \\
\hline Serpophaga subcristata & & & & & $\mathrm{x}$ & 0,56 & & \\
\hline Myiarchus swainsoni & 0,02 & 0,56 & & & & & & \\
\hline Pitangus sulphuratus & 0,02 & 1,00 & & & 0,06 & 1,00 & $\mathrm{x}$ & 1,00 \\
\hline Machetornis rixosa & & & & & & & $\mathrm{x}$ & 1,00 \\
\hline Myiodynastes maculatus & & & 0,03 & 0,56 & 0,02 & 0,44 & & \\
\hline Tyrannus melancholicus & & & & & 0,04 & 0,56 & $\mathrm{x}$ & 0,56 \\
\hline Tyrannus savana & & & & & & & $\mathrm{x}$ & 0,44 \\
\hline Lathrotriccus euleri & 0,05 & 0,56 & 0,10 & 0,33 & & & & \\
\hline Satrapa icterophrys & & & & & & & $\mathrm{x}$ & 0,22 \\
\hline \multicolumn{9}{|l|}{ VIREONIDAE } \\
\hline Cyclarhis gujanensis & 0,06 & 1,00 & $\mathrm{x}$ & 0,78 & 0,04 & 0,56 & & \\
\hline Vireo olivaceus & $\mathrm{x}$ & 0,11 & & & & & & \\
\hline \multicolumn{9}{|l|}{ HIRUNDINIDAE } \\
\hline Pygochelidon cyanoleuca & & & & & & & $\mathrm{x}$ & 1,00 \\
\hline Progne tapera & & & & & & & $\mathrm{x}$ & 0,56 \\
\hline Progne chalybea & & & & & & & $\mathrm{x}$ & 0,56 \\
\hline Tachycineta leucorrhoa & & & & & & & $\mathrm{x}$ & 0,44 \\
\hline \multicolumn{9}{|l|}{ TROGLODYTIDAE } \\
\hline Troglodytes musculus & & & & & 0,06 & 0,78 & $\mathrm{x}$ & 1,00 \\
\hline \multicolumn{9}{|l|}{ TURDIDAE } \\
\hline Turdus rufiventris & 0,08 & 1,00 & 0,03 & 0,44 & 0,06 & 1,00 & $\mathrm{x}$ & 0,89 \\
\hline Turdus leucomelas & & & & & 0,06 & 0,78 & $\mathrm{x}$ & 0,67 \\
\hline Turdus amaurochalinus & & & & & & & $\mathrm{x}$ & 1,00 \\
\hline Turdus albicollis & 0,06 & 1,00 & 0,03 & 0,22 & & & & \\
\hline \multicolumn{9}{|l|}{ COEREBIDAE } \\
\hline Coereba flaveola & 0,03 & 1,00 & 0,03 & 0,56 & $\mathrm{x}$ & 0,89 & $\mathrm{x}$ & 1,00 \\
\hline \multicolumn{9}{|l|}{ THRAUPIDAE } \\
\hline Saltator similis & 0,01 & 0,78 & & & & & $\mathrm{x}$ & 0,22 \\
\hline Lanio cucullatus & $\mathrm{x}$ & 0,11 & & & & & & \\
\hline Tangara sayaca & 0,01 & 1,00 & & & 0,08 & 0,78 & $\mathrm{x}$ & 0,44 \\
\hline Tangara preciosa & 0,02 & 0,67 & & & 0,02 & 0,67 & $\mathrm{x}$ & 0,22 \\
\hline Pipraeidea melanonota & & & & & $\mathrm{x}$ & 0,11 & & \\
\hline Pipraeidea bonariensis & & & & & & & $\mathrm{x}$ & 0,22 \\
\hline \multicolumn{9}{|l|}{ EMBERIZIDAE } \\
\hline Zonotrichia capensis & & & & & $\mathrm{x}$ & 0,56 & $\mathrm{x}$ & 0,67 \\
\hline Poospiza lateralis & & & $\mathrm{x}$ & 0,44 & & & $\mathrm{x}$ & 0,33 \\
\hline Sicalis flaveola & & & & & & & $\mathrm{x}$ & 1,00 \\
\hline Sporophila caerulescens & & & & & & & $\mathrm{x}$ & 0,11 \\
\hline
\end{tabular}


TABELA 1: Continuação ...

TABLE 1: Continued ...

\begin{tabular}{|c|c|c|c|c|c|c|c|c|}
\hline \multirow{2}{*}{ FAMÍLIA/Espécie } & FS & \multicolumn{3}{|c|}{ PE } & \multirow{2}{*}{$\begin{array}{c}\text { PP } \\
\text { abundância }\end{array}$} & \multicolumn{3}{|c|}{$\mathrm{AB}$} \\
\hline & abundância & FO & abundância & FO & & FO & abundância & FO \\
\hline \multicolumn{9}{|l|}{ PARULIDAE } \\
\hline Parula pitiayumi & 0,06 & 1,00 & 0,05 & 1,00 & 0,12 & 1,00 & $\mathrm{x}$ & 0,78 \\
\hline Geothlypis aequinoctialis & & & & & & & $\mathrm{x}$ & 0,33 \\
\hline Basileuterus culicivorus & 0,07 & 1,00 & $\mathrm{x}$ & 0,67 & 0,08 & 0,67 & & \\
\hline Basileuterus leucoblepharus & 0,13 & 1,00 & 0,21 & 1,00 & 0,02 & 0,89 & & \\
\hline \multicolumn{9}{|l|}{ ICTERIDAE } \\
\hline Icterus pyrrhopterus & $\mathrm{x}$ & 0,11 & & & & & & \\
\hline Agelaioides badius & & & & & & & $\mathrm{x}$ & 0,78 \\
\hline \multicolumn{9}{|l|}{ FRINGILIDAE } \\
\hline Euphonia chlorotica & 0,01 & 1,00 & 0,03 & 0,67 & 0,02 & 0,67 & $\mathrm{x}$ & 0,67 \\
\hline Euphonia pectoralis & 0,01 & 0,56 & & & & & & \\
\hline \multicolumn{9}{|l|}{ ESTRILDIDAE } \\
\hline Estrilda astrild & & & & & & & $\mathrm{x}$ & 0,11 \\
\hline
\end{tabular}

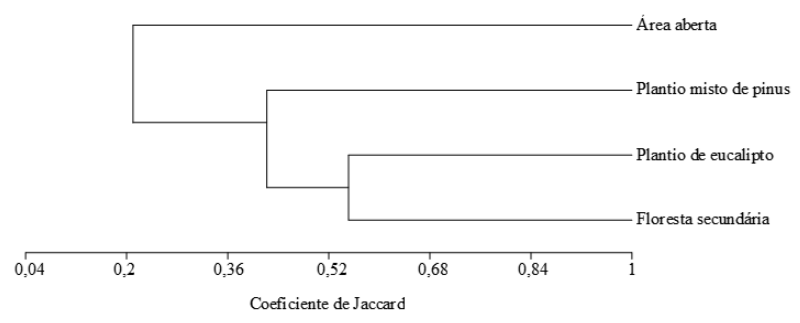

FIGURA 2: Similaridade na composição da avifauna de quatro fitofisionomias amostradas em propriedade rural do município de Viamão - RS, de maio a novembro de 2006.

FIGURE 2: Similarity in the aviafuna composition of four vegetation physiognomies studied in a rural area of the city of Viamão, state of Rio Grande do Sul, from May to November 2006.

florestais (Tabela 1), tais como Nothura maculosa, Amazonetta brasiliensis, Mycteria americana, Milvago chimango, Myiopsitta monachus, Colaptes campestris, Furnarius rufus, Machetornis rixosa, Tyrannus savana, Tachycineta leucorrhoa, Progne spp.e Estrildaastrild, por exemplo. Já afloresta nativa secundária, o plantio de eucalipto e o plantio misto de pinus apresentaram maior similaridade quanto à composição da avifauna, uma vez que compartilham espécies associadas a ambientes florestais. Todavia, as espécies comuns a esses ambientes são, em sua maioria, generalistas e tolerantes à ação antrópica, a exemplo de Hylocharis chysura, Veniliornis spilogaster, Colaptes melanochloros, Cranioleuca obsoleta, Camptostoma obsoletum, Turdus rufiventris, Coereba flaveola, Parula pitiayumi e Euphonia chlorotica.

No que se refere ao conjunto das espécies registradas, foram reconhecidas sete categorias tróficas (Figura 3). As espécies insetívoras foram as mais representativas em todos os ambientes amostrados, seguidas pelas espécies frugívoras (exceto na área aberta) e onívoras. Representantes carnívoros apresentaram baixas porcentagens em todas as fitofisionomias estudadas.

\section{DISCUSSÃO}

O registro de aproximadamente $14 \%$ da avifauna presente no Rio Grande do Sul (BENCKE et al., 2010) em uma área de pequenas dimensões não deve ser desprezado. Embora a riqueza de espécies tenha sido inferior àquela encontrada por Efe et al. (2001) no parque Saint Hilaire, em Viamão, equivalente a 133 espécies, assim como por Scherer et al. (2005), em oito parques de Porto Alegre (170 espécies), deve-se ponderar que o tamanho das áreas estudadas pelos autores foi consideravelmente maior que a deste estudo e o esforço amostral foi superior ao despendido neste. Em relação a outras 


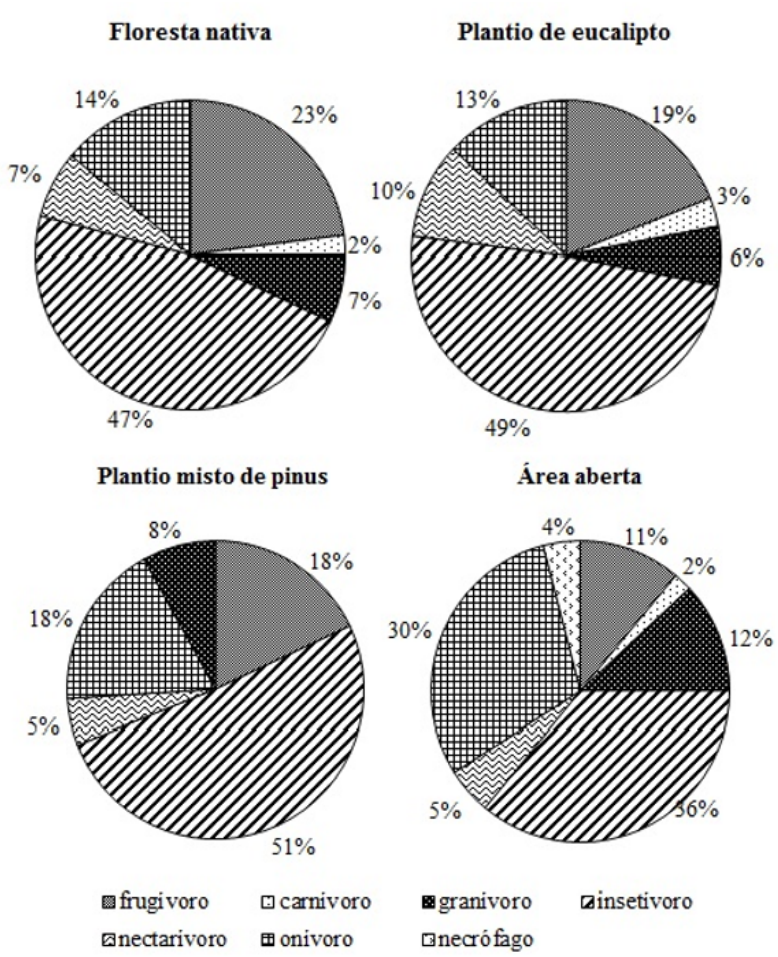

FIGURA 3: Guildas tróficas das espécies registradas nos distintos ambientes amostrados em propriedade rural do município de Viamão - RS, de maio a novembro de 2006.

FIGURE 3: Trophic guilds of birds registered in the different environments studied in a rural area of the city of Viamão, state of Rio Grande do Sul, from May to November 2006.

investigações, entretanto, o número de espécies encontradas pode ser considerado expressivo. Voss (1981) registrou, de junho a novembro de 1979, 70 espécies no Jardim Botânico de Porto Alegre, em uma área de 43 ha. Desde este levantamento pioneiro, estudos complementares foram realizados por vários pesquisadores, chegando-se, atualmente, à riqueza de 126 espécies (FZB-RS, 2004). No Parque Farroupilha (30,7 ha aproximadamente), Sander e Voss (1982) registraram, em um ano de amostragem, 46 espécies. Mendonça-Lima e Fontana (2000) constataram, no Porto Alegre Country Clube (47 ha), um total de 63 espécies durante um ano de estudo, ao longo de $75 \mathrm{~h}$ de observações. Por conseguinte, comparativamente aos resultados encontrados na literatura, a área de estudo é consideravelmente diversa, uma vez que além de apresentar menores dimensões, o esforço amostral empreendido foi inferior ou semelhante ao dos estudos mencionados anteriormente.

A floresta nativa, embora tenha apresentado frequência absoluta de indivíduos maior que no plantio de eucalipto e no plantio misto de pinus, compartilhou $55 \%$ e $40 \%$ das espécies, respectivamente, com estes. Atribui-se a ocorrência de muitas espécies nesses plantios ao complexo mosaico ambiental que compõe a área de estudo e ao remanescente florestal nativo presente na área. A ocorrência de espécies como Ortalis guttata, Lepidocolaptes falcinellus, Syndactyla rufosuperciliata, Elaenia parvirostris, Phylloscartes ventralis, Tolmomyas sulphurescens, Basileuterus culicivorus e Basileuterus leucoblepharus nas três fitofisionomias, deve-se, fundamentalmente, à existência do remanescente de vegetação nativa, que provavelmente atua como área fonte de indivíduos às regiões adjacentes (SANTOS e CADEMARTORI, 2010).

A maior abundância e frequência de ocorrência de espécies na floresta nativa secundária, principalmente daquelas seletivas quanto à escolha de habitats, como Crypturellus obsoletus, Piculus aurulentus, Sclerurus scansor, Dendrocolaptes platyrostris, Lepidocolaptes falcinellus e Euphonia pectoralis, por exemplo, demonstra a importância desse ambiente para a manutenção de espécies florestais. As demais áreas apresentaram táxons generalistas, com abundâncias e frequências de ocorrência, em sua maioria, mais baixas em comparação à floresta nativa. Espécies comuns ou com frequências de ocorrência elevadas nas áreas de plantio, como Milvago chimachima, Aramides saracura, Troglodytes musculus, Coereba flaveola ou Euphonia chlorotica, são generalistas quanto à escolha de hábitats e usualmente encontradas em qualquer área alterada pela ação antrópica (BELTON, 1994; WILLIS, 2000).

O maior índice de diversidade de Shannon foi registrado no remanescente de vegetação nativa, seguido pelo plantio misto de pinus e pelo plantio de eucalipto. Esses resultados apontam a floresta nativa como o ambiente que apresentou maior relação entre riqueza e equitatividade de espécies. Este fato pode ser atribuído à grande quantidade $\mathrm{e}$ variedade de recursos presentes nessa fitofisionomia, comparativamente aos plantios, o que contribui para a ocorrência de táxons adaptados a diferentes estratégias de alimentação e forrageamento.

No que diz respeito à similaridade entre os ambientes, a floresta nativa, o plantio de eucalipto 
e o plantio misto de pinus formam um grupo que compartilha cerca de $40 \%$ das espécies de aves. Entretanto, o plantio misto de pinus, por consistir em uma fitofisionomia bastante heterogênea, apresenta composição de espécies diversificada, diferenciando-se, deste modo, da floresta nativa e do plantio de eucalipto. Por não se tratar de um plantio comercial e sim de uma área destinada ao lazer, na qual o pinus foi plantado há mais de 50 anos com fins ornamentais, o espaçamento entre os indivíduos arbóreos é maior. Assim, ao longo do tempo, muitas espécies vegetais nativas puderam se estabelecer na área. Além disso, o local apresenta uma área úmida com muitas espécies vegetais associadas a ambientes palustres. Por esse motivo, muitas espécies de aves não associadas a ambientes florestais utilizam a área, como Nycticorax nycticorax e Syrigma sibilatrix, por exemplo. Por se tratar de uma área de dimensões reduzidas, é mais afetada pelo efeito de borda. Consequentemente, muitas espécies de áreas adjacentes (como as da área aberta, por exemplo) ocorrem com certa frequência no plantio misto de pinus, tais como Milvago chimachima, Columbina picui, Zenaida auriculata, Troglodytes musculus e Zonotrichia capensis.

A análise de guildas tróficas demonstrou que $23 \%$ das espécies registradas na floresta secundária são frugívoras, importantes à dispersão de sementes e na regeneração de ambientes (SICK e PACHECO, 1997; PIZO, 2001). O maior percentual de espécies onívoras e de insetívoras generalistas constatado no plantio de eucalipto, no plantio misto de pinus e na área aberta expressa o considerável grau de alteração desses habitats. Por outro lado, a maior proporção de frugívoros, além da presença de carnívoros e nectarívoros, formas mais especializadas quanto à dieta, especialmente no fragmento de floresta nativa, evidencia a melhor qualidade do ambiente em questão. Motta-Júnior (1990) menciona que ambientes com altos índices de degradação ambiental apresentam número crescente de aves onívoras e, possivelmente, insetívoras menos especializadas, transcorrendo situação oposta no que tange às frugívoras e insetívoras mais especializadas.

Os resultados obtidos neste estudo demonstram, para determinadas espécies, um padrão de preferência por habitats florestais que conservam, ainda que parcialmente, suas características originais. Crypturellus obsoletus, Piculus aurulentus, Sclerurus scansor, Dendrocolaptes platyrostris, Conopophaga lineata, Myiarchus swainsoni, Chiroxiphia caudata e Euphonia pectoralis foram registradas exclusivamente em ambiente de floresta nativa secundária. De acordo com a literatura (WILLIS, 1979; ALEIXO e VIELLIARD, 1995; SICK e PACHECO, 1997; ALEIXO, 2001; ANJOS, 2001), representantes da família Tinamidae e Dendrocolaptidae, como Crypturellus obsoletus e Dendrocolaptes platyrostris, respectivamente, são seletivas quanto à escolha de habitats, desaparecendo de ambientes fragmentados ou muito alterados. Anjos (2001) registrou diminuição no número de contatos de Crypturellus obsoletus com a redução do tamanho da área de fragmentos e manchas florestais na bacia do Rio Tibagi, no estado do Paraná, indicando que essa espécie é diretamente atingida pela fragmentação. Aleixo e Vielliard (1995) relatam a extinção da família Dendrocolaptidae na Mata de Santa Genebra, no estado de São Paulo, tomando por base o estudo de Willis (1979) que lá havia registrado cinco espécies de dendrocolaptídeos, entre as quais Sittasomus griseicapillus e Dendrocolaptes platyrostris. Entre as causas da extinção dessa família (bem como de espécies pertencentes a outras famílias) estão o grau de isolamento do fragmento, o histórico de perturbação da área e a incapacidade de utilização do habitat matriz (canavial) por parte de espécies florestais mais especializadas (ALEIXO e VIELLIARD, 1995; ALEIXO, 2001). Neste estudo, Sittasomus griseicapillus e Dendrocolaptes platyrostris foram registradas no interior do fragmento florestal nativo, evitando suas bordas. É provável que tais espécies façam uso do habitat matriz para deslocamentos entre fragmentos adjacentes de maiores dimensões. Fontana (2005) considera Dendrocolaptes platyrostris como em perigo de extinção em Porto Alegre, uma vez que, por ser associada à Mata Atlântica (ALEIXO, 2001), não dispõe atualmente de locais apropriados de vida na capital gaúcha a não ser pelos registros mencionados pela autora no Morro Santana. É possível que essa espécie ainda possua populações viáveis em remanescentes da região sul na capital gaúcha, como na Reserva Estadual do Lami (distante cerca de $6 \mathrm{~km}$ da área de estudo) ou no Parque Estadual de Itapuã.

Os resultados obtidos evidenciam a importância de estudos concernentes à avifauna em ambientes rurais nas cercanias de grandes cidades. Tais ambientes podem servir de refúgio às espécies, uma vez que seus habitats naturais diminuem em ritmo acelerado (WILLIS, 1979; 2000). A área 
estudada fica a cerca de $6 \mathrm{~km}$ do limite municipal entre Porto Alegre e Viamão. As ocorrências de algumas espécies consideradas quase extintas em Porto Alegre, como Thalurania glaucopis e Dendrocolaptes platyrostris, além de outras cujos registros vêm declinando na capital gaúcha, como Ortalis guttata, Tolmomyas sulphurescens e Tangara preciosa (FONTANA, 2005), indicam que, mediante ações adequadas de manejo, há possibilidade de conservação dessas espécies na região. Outra espécie que merece destaque é Piculus aurulentus, considerada quase ameaçada em nível global (IUCN, 2011) devido à fragmentação e exploração florestal, mas comum na área de estudo.

A fragmentação florestal pode levar à perda de diversidade biológica. Tal evento provavelmente ocorreu em um passado não muito distante na região, fato este evidenciadopela pesquisa deFontana(2005). Assim, um dos principais aspectos que deve nortear novas investigações, em escala local e regional, é o estudo da composição e tamanhos populacionais de espécies de aves nos remanescentes florestais. Populações pequenas não são viáveis em longo prazo, pois o isolamento geográfico pode acarretar em consequências desfavoráveis decorrentes da fixação de características deletérias, causada pelo processo de endogamia (BRITTO e FERNANDEZ, 2000). Por conseguinte, estudos de monitoramento das populações forneceriam importantes subsídios a ações de manejo e conservação da avifauna. Uma das prioridades seria investigar a dinâmica populacional de Piculus aurulentus e Dendrocolaptes platyrostris, espécies mais seletivas quanto à escolha de habitats, dependentes de cobertura florestal mais expressiva e certamente subamostradas em levantamentos anteriores.

\section{CONCLUSÃO}

A heterogeneidade de fitofisionomias que caracteriza a região de estudo, incluindo áreas úmidas, campos, florestas nativas e plantios exóticos, favoreceu uma avifauna diversificada. As áreas florestais foram similares no que concerne à composição de espécies de aves. No entanto, o remanescente de floresta nativa apresentou maior abundância e frequência de ocorrência de aves seletivas quanto à escolha de habitats, diferentemente das áreas de plantio, nas quais espécies seletivas foram menos frequentes ou ausentes.

Os resultados obtidos demonstram a importância de áreas com vegetação nativa para a manutenção de espécies de aves seletivas quanto à escolha de habitats e suscitam a necessidade de novos estudos na área e em fragmentos próximos, com o objetivo de se conhecer melhor a composição e a abundância de espécies em ambientes degradados.

A preocupação com a conservação deve ser o principal foco no desenvolvimento de ações de restauração e manejo da vida silvestre. O monitoramento e o manejo de populações em áreas rurais podem ser importantes estratégias para a conservação da avifauna local e regional.

\section{AGRADECIMENTOS}

À comunidade dos Irmãos de Nossa Senhora de Maria Auxiliadora, pela hospedagem, apoio a este estudo e, principalmente, pela amizade; ao Unilasalle, pelo transporte fornecido e concessão de BIC; aos amigos Matheus e Camila Saraiva, pelo auxílio em campo; ao Prof. Sydney Sabedot e à Priscila Meneghetti Eger, pelos dados referentes às dimensões da área de estudo.

\section{REFERÊNCIAS BIBLIOGRÁFICAS}

ACCORDI, I. A. Avifauna de três sítios contíguos na zona urbana do município de Canoas, RS. Acta Biologica Leopoldensia, v. 23 , n. 1, p. 59-68, 2001.

ALEIXO, A. Conservação da avifauna da Floresta Atlântica: efeitos da fragmentação e a importância de florestas secundárias. In: ALBUQUERQUE, J. L. B. et al. (Eds.). Ornitologia e conservação: da ciência às estratégias. Tubarão: Unisul, 2001. p. 199-206.

ALEIXO, A.; VIELLIARD, J. M. E. Composição e dinâmica da avifauna da Mata de Santa Genebra, Campinas, São Paulo, Brasil. Revista Brasileira de Zoologia, v. 12, n. 3, p. 493-511, 1995.

ANJOS, L. dos. Comunidades de aves florestais: implicações na conservação. In: ALBUQUERQUE, J. L. B. et al. (Eds.). Ornitologia e conservação: da ciência às estratégias. Tubarão: Unisul, 2001. p. 17-38.

BELTON, W. Aves do Rio Grande do Sul: distribuição e biologia. São Leopoldo: UNISINOS, 1994. $584 \mathrm{p}$.

BENCKE et al. Revisão e atualização da lista das aves do Rio Grande do Sul, Brasil. Iheringia, Sér. Zool., v. 100, n. 4, p. 519-556, 2010.

BENCKE, G. A. et al. Aves. In: FONTANA, C. S.; BENCKE, G. A.; REIS, R. E. (Eds.). Livro 
Vermelho da fauna ameaçada de extinção no Rio Grande do Sul. Porto Alegre: EDIPUCRS, 2003. p. $189-479$.

BRITO, D.; FERNANDEZ, F. A. S. Dealing with extinction is forever: understanding the risks faced by small populations. Ciência e Cultura Jounal of the Brazilian Association for the Advanced of Science, v. 52, n. 3, p. 161-170, 2000.

CBRO, Comitê Brasileiro de Registros Ornitológicos. Listas das aves do Brasil. 2011. 10. ed. Disponível em: <http://www.cbro.org.br>. Acesso em: 28 ago. 2012.

EFE, M. A. et al. Inventário e distribuição da avifauna do Parque Saint'Hilaire, Viamão, Rio Grande do Sul, Brasil. Tangara, v. 1, n. 1, p. 12-25, 2001.

FONTANA, C. S. A ornitofauna em Porto Alegre no século XX: status de ocorrência e conservação.

Comunicações do Museu de Ciências - PUCRS, Série Zoologia, v. 18, n. 2, p. 73-212, 2005.

FZB-RS, Fundação Zoobotânica do Rio Grande do Sul. Plano Diretor do Jardim Botânico de Porto Alegre. Publicações Avulsas FZB, v. 12. Porto Alegre: FZB-RS, 2004.

GIMENES, R. G.; ANJOS, L. Efeitos da fragmentação florestal sobre as comunidades de aves. Acta Scientiarum, Biological Sciences, v. 25, n. 2, p. 391-402, 2003.

GRILLO, H. C. Z.; BENCKE, G. A. Aves do novo campus da Universidade do Vale do Rio dos Sinos, UNISINOS, São Leopoldo, RS. Acta Biologica Leopoldensia, v. 17, n. 1, p. 123-145, 1995.

IUCN 2011. IUCN Red List of Threatened Species. Version 2011.2. Disponível em: <http://www. iucnredlist.org >. Acesso em: 11 maio 2012.

KOVACH, W. L. MVSP: a multivariate statistical package for windows, ver. 3.1. Pentraeth, Wales, U.K.: Kovach Computing Services, 1999.

MENDONÇA-LIMA, A.; FONTANA, C. S. Composição, freqüência e aspectos biológicos da avifauna no Porto Alegre Country Clube, Rio Grande do Sul. Ararajuba, v. 8, n. 1, p. 1-8, 2000. MATARAZZO-NEUBERGUER, W. M. Comunidades de aves de cinco parques e praças da Grande São Paulo, Estado de São Paulo. Ararajuba, v. 3, p. 13-19, 1995.

MOTTA-JÚNIOR, J. C. Estrutura trófica e composição de três habitats terrestres na região central do Estado de São Paulo. Ararajuba, v. 1, p. 65-71, 1990.

PIZO, M. A. A conservação das aves frugívoras. In: ALBUQUERQUE, J. L. B. et al. (Eds.). Ornitologia e conservação: da ciência às estratégias. Tubarão: Unisul, 2001. p. 49-59.

SANDER, M.; VOSS, W. A. Aves livres observadas no Parque Farroupilha, Porto Alegre, Rio Grande do Sul, Brasil. Pesquisas, Série Zoologia, n. 33, p. 3-16, 1982.

SANTOS, M. F. B.; CADEMARTORI, C. V. Estudo comparativo da avifauna em áreas verdes urbanas da região metropolitana de Porto Alegre, sul do Brasil. Biotemas, v. 23, n. 1, p. 181-195, 2010.

SANTOS, M. F. B.; CADEMARTORI, C. V. Contribuição ao conhecimento da avifauna do município de Araricá, Rio Grande do Sul. Biotemas, v. 20, n. 2, p. 41-48, 2007.

SCHERER, A. et al. Estrutura trófica da Avifauna em oito parques da cidade de Porto Alegre, Rio Grande do Sul, Brasil. Ornithologia, v. 1, n. 1, p. 25-32, 2005.

SCHERER, J. S. M. et al. Estudo da avifauna associada à área úmida situada no Parque Mascarenhas de Moraes, zona urbana de Porto Alegre (RS). Biotemas, v. 19, n. 1, p. 107-110, 2006.

SICK, H.; PACHECO, J. F. (Coord.). Ornitologia Brasileira. Rio de Janeiro: Nova Fronteira, 1997. $862 \mathrm{p}$.

TAMPSON, V. E. Lista comentada das espécies de aves registradas para o morro do Espelho, São Leopoldo, Rio Grande do Sul, Brasil (1983-1988). Acta Biologica Leopoldensia, v. 12, n. 1, p. 19-37, 1990.

WILLIS, E. O. The composition of avian communities in remanescent woodlots in Southern Brazil. Papéis Avulsos de Zoologia, v. 33, n. 1, p. 1-25, 1979.

WILLIS, E. O. Ranking urban avifaunas (Aves) by number of localities per species in São Paulo, Brazil.

Iheringia, Sér. Zool., v. 88, p. 139-146, 2000.

WILKINSON, L. Systat 11. San José, USA: Systat Software Inc., 2004.

VOSS, W. A. Aves de Porto Alegre, Rio Grande do Sul. III - Aves observadas no Jardim Botânico da Fundação Zoobotânica do Rio Grande do Sul. Acta Biologica Leopoldensia, v. 3, n. 1, p. 81-94, 1981. 